\title{
Uso do equipamento para controle da incontinência anal no paciente com dermatite: relato de caso
}

\author{
Use of the device for control of anal incontinence in patients with dermatitis: \\ case report
}

\author{
Uso del equipamiento de controle de la incontinencia anal en paciente con \\ dermatitis: relato de caso
}

Ana Patrícia de Cerqueira Greco ${ }^{1}$, Andressa de França Guimarães ${ }^{1}$, Taíse Santos do Nascimento ${ }^{1}$

\begin{abstract}
RESUMO
A dermatite associada à incontinência (DAl) é definida como lesões de pele ocasionadas por umidade, sendo frequentes em pacientes com incontinência anal (IA) e/ou urinária, nos quais ocorre contato direto da pele com a umidade. O tratamento e o manejo, por vezes, são obstáculos para o enfermeiro. O objetivo deste artigo foi descrever a utilização do equipamento de controle de IA e demonstrar sua eficácia no manejo de pacientes com DAl. Trata-se de um estudo qualitativo, descritivo, retrospectivo, do tipo estudo de caso. O paciente selecionado esteve internado na unidade de terapia semi-intensiva de um hospital privado, em Salvador (BA). O artigo relata a evolução e o tratamento da DAl, desde a indicação do uso do equipamento até sua retirada. A coleta e a análise dos dados foram realizadas com base nos registros sobre a utilização do equipamento em prontuário eletrônico, exame físico do paciente e evoluções de enfermagem. A pesquisa obedeceu aos princípios da Resolução nº 466/12 do Conselho Nacional de Saúde (CNS), após aprovação no Comitê de Ética em Pesquisa (n 15/2014). A utilização do equipamento foi efetiva para o tratamento da DAl, evitando a progressão da lesão, diminuindo a dor e proporcionando conforto ao paciente, mostrando-se, portanto, eficaz pela resolutividade. Percebe-se a necessidade de maior conhecimento da equipe multiprofissional quanto ao equipamento e de maior número de pesquisas científicas com essa temática.
\end{abstract}

DESCRITORES: Dermatite. Enfermagem. Equipamentos e provisões. Estomaterapia.

\begin{abstract}
Dermatitis associated with incontinence (DAI) is defined as skin lesions caused by humidity, frequent in patients with fecal and/or urinary incontinence, in which there is direct skin contact with moisture. The treatment and management of these patients are sometimes obstacles for nurses. This work aimed to describe the use of fecal incontinence control devices and demonstrate their efficacy in patients with DAl. This is a qualitative research, descriptive, retrospective case study. The selected patient was admitted in the semi-intensive care unit of a private hospital in Salvador, Bahia. The study reports on the evolution and treatment of DAl, from the indication of the use of the device to its removal. Data collection and analysis were based on records of the use of the electronic medical device, clinical evaluation and physical examination of the patient and nursing progress. The research followed the principles of Resolution $n^{\circ} 466 / 12$ of the National Health Council (CNS), after approval by the Research Ethics Committee ( $n^{\circ}$ 15/2014). The use of the device was effective in the treatment of DAl injury, preventing its progression, reducing pain and providing comfort to the patient, being therefore effective for solving this issue. We see the need for greater knowledge of the multidisciplinary team about this device and more scientific research on this subject.
\end{abstract}

DESCRIPTORS: Dermatitis. Nursing. Equipment and supplies. Enterostomal Therapy. 


\section{RESUMEN}

Dermatitis asociada con la incontinencia (DAI), se define como lesiones en la piel causadas por la humedad, frecuente en pacientes con incontinencia urinaria y/o fecal, en la que haya un contacto directo de la piel con la humedad. El tratamiento y manejo de estos pacientes a veces son obstáculos para el enfermero(a), el objetivo de este artículo, fue describir la utilización del equipo de control de IA y demostrar su eficacia en el manejo de pacientes con DAI. Se trata de un estudio cualitativo, descriptivo, retrospectivo, del tipo de estudio de caso. El paciente seleccionado estuvo hospitalizado en la unidad de terapia semi- intensiva de un hospital privado, en Salvador de Bahía. El articulo relata la evolución y el tratamiento de la DAI desde la indicación del uso del equipo hasta su retiro, la recolección y analisis de los datos fueron realizados con base en los registros sobre la utilización del equipo en fichas electrónicas, examen físico del paciente y evoluciones de enfermería. La investigación obedeció a los principios de resolución N. 466 /12, del consejo nacional de salud (CNS), después de la aprobación en el comité de ética en investigación (N. 15/ 2014). La utilización del equipo fue efectiva para el tratamiento de la DAl, evitando la progresión de la lesión, disminuyendo el dolor y proporcionando confort al paciente, mostrándose eficaz por la resolución. Se percibe la necesidad de tener mayor conocimiento por parte del equipo multi-profesional con relación al equipo y de realizar mayor número de investigaciones científicas con esa temática.

DESCRIPTORES: Dermatitis. Enfermería. Equipos y suministros. Estomaterapia.

\section{INTRODUÇÃO}

O desenvolvimento de lesões de pele associadas à presença de umidade é comum na prática da enfermagem, sendo muitas vezes confundidas pela equipe com lesões por pressão ${ }^{1}$. A diferenciação pelo enfermeiro é fundamental, pois as medidas preventivas e os cuidados são diferenciados ${ }^{2}$.

A dermatite associada à incontinência (DAI) é definida como lesões de pele ocasionadas por umidade frequente em pacientes com incontinência anal (IA) e/ou urinária. $\mathrm{O}$ contato prolongado com urina e fezes aumenta a incidência desse tipo de lesão, que se caracteriza por inflamação da pele com aspecto inicial de hiperemia, podendo evoluir para ruptura da pele, denominada ulceração ${ }^{2}$. O principal fator de risco para a DAI é a presença de incontinência ${ }^{3}$.

A IA é um dos fatores associados ao surgimento da DAI, sendo definida pela International Continence Society como perda involuntária de fezes ou gases, em qualquer faixa etária, após a aquisição do controle esfincteriano ${ }^{4}$. A prevalência da DAI é pouco conhecida, e os dados internacionais de prevalência de IA mostram valores que vão de 1,9 a 21,3\% da população, elevando-se com o aumento da faixa etária. Um estudo com pacientes críticos de uma unidade de terapia intensiva encontrou prevalência de $15,27 \%$.

Assim, o presente estudo teve como questão norteadora: como o equipamento de controle da IA pode auxiliar o enfermeiro no manejo de pacientes com DAI? O objetivo geral foi descrever a utilização do equipamento de controle de IA e demonstrar sua eficácia no manejo de pacientes com DAI.

\section{MÉTODOS}

Estudo qualitativo, descritivo, retrospectivo, com paciente internado na unidade de terapia semi-intensiva de um hospital privado, na cidade de Salvador, Bahia. A coleta de dados ocorreu de 2 a 21 de junho de 2014, utilizando registros em prontuário eletrônico, evolução clínica e evoluções de registros de enfermagem. A pesquisa obedeceu à Resolução no 466/12, com aprovação do Comitê de Ética em Pesquisa (no 15/2014) e assinatura pelo responsável do Termo de Consentimento Livre e Esclarecido ${ }^{6}$.

\section{RESULTADOS E DISCUSSÃO}

Paciente de 31 anos, restrito ao leito, com síndrome cognitiva secundária à doença de Wilson, uma patologia genética e degenerativa que provoca alteração no metabolismo de cobre e comprometimento neurológico e psiquiátrico ${ }^{7}$.

O paciente foi atendido por insuficiência respiratória aguda e transferido para a unidade semi-intensiva. Apresentou dermatite extensa em regiões perianal, interglútea e glútea ocasionada por umidade decorrente de IA. 
Medidas protetoras como higiene do local, trocas frequentes de fralda e uso de creme barreira não foram suficientes para impedir o aparecimento da DAI, uma vez que esta pode ocorrer em razão do contato das fezes líquidas e semilíquidas com a pele do paciente acamado ${ }^{8,9}$. O indivíduo apresentava alto risco de desenvolver lesão por pressão, na aplicação da escala de Braden.

Como medida terapêutica para controle da DAI, foi proposta a utilização do equipamento de controle de IA, com o objetivo de limitar a dispersão das fezes, reduzindo o seu contato com a pele, evitando a irritação e a maceração, preservando a função de barreira cutânea e mantendo, sempre que possível, um $\mathrm{pH}$ ácido ${ }^{10}$.

A indicação ocorreu a partir de protocolo assistencial institucional, o qual define o uso em pacientes que apresentam três episódios de dejeções líquidas ou semilíquidas por mais de dois dias consecutivos, com alto risco de lesão por pressão identificado e/ou com a integridade da pele prejudicada. Modificações na dieta e utilização de simbióticos são práticas adotadas pela equipe médica e, caso o paciente não obtenha melhora da diarreia após 72 horas, o equipamento será indicado pelo enfermeiro e/ou médico.

O equipamento de controle de IA é utilizado no Brasil desde 2007. O cateter é posicionado na cavidade retal e retido por um balão inflado com $45 \mathrm{~mL}$ de água ou soro fisiológico, exercendo pressão baixa na mucosa retal e minimizando os riscos de lesões graves como isquemia e necrose ${ }^{10}$.

Um estudo observacional $(n=15)$ demonstrou a eficácia do equipamento de IA no manejo do tratamento e no desvio das fezes em pacientes queimados, com média de permanência do cateter de 22,5 $\pm 5,7$ dias, não sendo encontradas complicações graves associadas ao uso do equipamento, exceto por erosão superficial do reto distal $(\mathrm{n}=2)^{11}$.

As contraindicações para utilização do equipamento são: intervenção cirúrgica na parte distal do intestino não grosso ou reto, lesão retal ou anal, estenose retal ou anal, mucosa distal prejudicada, tumores retais ou anais, hemorroidas severas ou impactação fecal. Pode-se utilizar o cateter no paciente por até 29 dias, com troca da bolsa coletora a cada 72 horas ou até atingir $900 \mathrm{~mL}$ de volume drenado ${ }^{10}$.

A inserção do cateter foi realizada pelo enfermeiro, sendo associado o uso de hidrocoloide em pó para a manutenção do meio livre de umidade a cada troca de fraldas. O paciente apresentava DAI extensa em regióes glútea, interglútea e perianal, com rupturas de pele difusas, exsudato serossanguinolento em pequeno volume e área perilesional hiperemiada.

O paciente teve episódios de fezes líquidas e semilíquidas com volume médio de $400 \mathrm{~mL} /$ dia nos 5 primeiros dias. Foram encontrados registros de enfermagem com relatos de melhora da DAI nas primeiras 24 horas de uso do equipamento. No quarto dia de uso, foi registrada melhora significativa, com epitelização das lesões nos glúteos.

No sexto e sétimo dias, registrou-se diminuição do volume fecal (250 e $150 \mathrm{~mL}$, respectivamente), com relatos de exteriorizações frequentes do equipamento ( $2-3$ vezes/dia) e fezes semilíquidas. A exteriorização é um evento comum e esperado, sendo necessário o reposicionamento do equipamento pelo enfermeiro de modo a garantir a manutenção e a evolução do processo cicatricial da DAI.

No oitavo dia verificou-se ausência de dejeções líquidas e semilíquidas, com epitelização total das lesões em regiões interglútea e perianal, optando-se pela retirada do equipamento e pela manutenção das áreas protegidas com creme barreira. O paciente obteve alta hospitalar com equipe multiprofissional domiciliar, recebendo orientações pelo enfermeiro sobre cuidados relacionados à manutenção da integridade da pele e à prevenção de DAI.

\section{CONCLUSÃO}

A utilização do equipamento contribuiu para a assistência qualificada, para o melhor manejo e para o tratamento da DAI por meio da redução do contato das fezes com a pele do paciente, da melhora da lesão, do maior conforto oferecido ao paciente e da redução dos custos adicionais com tratamentos prolongados.

Vale ressaltar que o tempo de cicatrização poderia ter sido reduzido se não houvesse a exteriorização do equipamento em alguns momentos, causando umidade local e exigindo recolocações frequentes. Observou-se a necessidade de maior conhecimento da equipe multiprofissional quanto à indicação do equipamento, aos cuidados de manutenção e ao melhor registro do volume das dejeções e da evolução da DAI, o que era esperado por se tratar de uma prática nova na instituição. $\mathrm{O}$ número reduzido de pesquisas científicas nacionais sobre o tema impossibilitou comparações do presente estudo com outros trabalhos que demonstrassem a utilização do equipamento. 


\section{REFERÊNCIAS}

1. National Pressure Ulcer Advesory Panel (NPUAP). NPUAP Pressure Injury Stages. 2016 [cited 2016 Aug 04] Available from: http://www.npuap.org/resources/educational-and-clinicalresources/npuap-pressure-injury-stages/

2. Bianchi J, Gomes TS. The dangers of faecal incontinence en the at-risk patient. Wounds Int [Internet]. 2012 [cited 2015 Apr 20];3:15-21. Available from: http://www. woundsinternational.com/journal-content/view/thedangers-of-faecal-incontinence-in-the-at-risk-patient

3. Cooper P. Incontinence-induced pressure ulcers. Nurs Res Care [Internet]. 2002. [cited 2015 May 20]:4(5):21621. Available from: http://www.magonlinelibrary.com/doi/ pdf/10.12968/nrec.2002.4.5.10385

4. Domansky RC, Borges EL. Manual para Prevenção de Lesões de Pele: recomendações baseadas em evidências. Rio de Janeiro: Rubio; 2012.

5. Abrams P, Andersson KE, Birder L, Brubaker L, Cardozo L, Chapple C, et al. Fourth international consultation on incontinence recommendations of the international scientific committee: evaluation and treatment of urinary incontinence, pelvic organ prolapse, and fecal incontinence. Neurourol Urodynam [Internet]. 2010 [cited 2015 Dec 15];29(1):213-40. Available from: http://www.ncbi.nlm.nih.gov/pubmed/20025020
6. Brasil. Resolução n. ${ }^{\circ}$ 466/12. Diretrizes e Normas Regulamentadoras de Pesquisas Envolvendo Seres Humanos. Brasília: Conselho Nacional de Saúde; 2012.

7. Prado ALC, Fonseca DC. Uma revisão sobre a doença de Wilson: Relato de Caso. Saúde [Internet]. 2004 [cited 2015 Jan 22];30(1-2):69-75. Available from: periodicos.ufsm.br/ revistasaude/article/viewFile/6396/

8. Baessa CEB, Meireles VC, Balan MAJ. Ocorrência de Dermatite Associada à Incontinência em Pacientes Internados na Unidade de Terapia Intensiva. Rev Estima. 2014;12(2):14-22.

9. Malaquias SG, Bachion MM, Nakatani AK. Risco de integridade da pele prejudicada em idosos hospitalizados. Cogitare Enferm [Internet]. 2008 [cited 2015 May 20];13(3):42836. Available from: http://revistas.ufpr.br/cogitare/article/ view/13040

10. Flexi Seal. Protocolo de Manejo Fecal. 2013.

11. Kement M, Acar HA, Barlas IS, Aksakal N, Gezen C, Düzci U, et al. Clinical Evaluation of a temporary fecal containment device for non-surgical fecal diversion in perineal burns. Turkish J Trauma Emergency Surgery [Internet]. 2011 [cited 2015 May 28];17(2):123-27. Available from: http://www.ncbi. nlm.nih.gov/pubmed/21644089 